\title{
Beneficial Effects of Grape Seed Extract on Malondialdehyde-Modified LDL
}

\author{
Atsushi SANO ${ }^{1}$, Riichiro UCHIDA ${ }^{1}$, Makoto SAITO ${ }^{1}$, Nobuhiko SHIOYA ${ }^{2}$, \\ Yoshika KOMORI ${ }^{2}$, Yasuo THO ${ }^{3}$ and Naotaka HASHIZUME ${ }^{4}$ \\ ${ }^{1}$ Research and Development Division, Kikkoman Corporation, 399 Noda, Noda, Chiba 278-0037, Japan \\ ${ }^{2}$ KSO Corporation, 1-2-3 Nishigahara, Kita-ku, Tokyo 114-0024, Japan \\ ${ }^{3}$ Aiwa Clinic, 1-590-1 Kawayanagi-cho, Koshigaya, Saitama 343-0827, Japan \\ ${ }^{4}$ Department of Health and Nutrition, Wayo Women's University, 2-3-1 Konodai, Ichikawa, \\ Chiba 272-8533, Japan \\ (Received September 14, 2006)
}

\begin{abstract}
Summary Following consecutive 12-wk administration of tablets containing 0, 200 or $400 \mathrm{mg}$ grape seed extract (calculated as proanthocyanidin) to 61 healthy subjects with LDL cholesterol (LDL-C) levels of 100 to $180 \mathrm{mg} / \mathrm{dL}$, effects of such treatment compared to administration of placebo tablets on malondialdehyde-modified LDL (MDA-LDL), representing one oxidized type of LDL, were investigated by a single blind method. MDA-LDL level in the $200 \mathrm{mg}$ (calculated as proanthocyanidin) group was significantly $(p=0.008)$ reduced compared to the basal level, $12 \mathrm{wk}$ after the start of administration. In the $400 \mathrm{mg}$ (calculated as proanthocyanidin) group, a significant decrease in MDA-LDL level compared to the basal level was found 6 and $12 \mathrm{wk}$ after the start of administration ( $6 \mathrm{wk}: p=0.015,12 \mathrm{wk}$ : $p=0.009)$. Subjects with high levels of MDA-LDL/ApoB (MDA-LDL/ApoB $\geq 100 \mathrm{mU} / \mathrm{mL}$ ) in the $200 \mathrm{mg}$ group showed significantly $(p=0.011)$ reduced MDA-LDL levels at $12 \mathrm{wk}$ after the start of administration. In the $400 \mathrm{mg}$ group, significant decreases in MDA-LDL level compared to the basal level were seen 6 and $12 \mathrm{wk}$ after the start of administration ( $6 \mathrm{wk}$ : $p=0.001,12 \mathrm{wk}: p<0.001)$; and at week 6 , significantly $(p=0.048)$ lower values were observed compared to those in patients who took placebo tablets (0 mg proanthocyanidin). In subjects demonstrating the least body weight changes during the test period (less than \pm $1.0 \mathrm{~kg})$ in the $400 \mathrm{mg}$ group, there was an increasing trend $(p=0.088)$ in adiponectin levels $12 \mathrm{wk}$ after the start of treatment. These results suggested that tablets containing grape seed extract exerted reducing effects on oxidized LDL, and might be useful in preventing lifestylerelated diseases such as arteriosclerosis.
\end{abstract}

Key Words grape seed extract, malondialdehyde-modified LDL, adiponectin, LDL oxidation

Previous epidemiologic studies showed that mortality due to arteriosclerosis-induced myocardial infarction was low in the French population despite their dietary habits characterized with a high intake of animal fat (the French paradox). It is suggested that the antioxidant effect of polyphenols contained in red wine which is frequently consumed by French people may account for such a paradox (1). So far, it has been shown that polyphenols in red wine demonstrates in vitro inhibition of oxidation of LDL-cholesterol (LDL-C) (2), and plasma LDL-C in subjects taking red wine through antioxidant effects (3). Among all polyphenols in red wine, proanthocyanidin is contained at the highest ratio, followed by catechin (4). It is also known that proanthocyanidin possesses the strongest antioxidant effect among polyphenols contained in red wine (5).

Oxidized LDL plays a central role in the occurrence of arteriosclerosis, and its progress in the body (6-9). In the present study, tablets containing proanthocyanidin

E-mail: asano@mail.kikkoman.co.jp which was highly purified from grape seeds as the main ingredient, were orally administered to adults of both sexes for $12 \mathrm{wk}$, in order to investigate effects on oxidized LDL and their safety (Study 1).

Recently, possible relationships between low concentrations of adiponectin and obesity (10), diabetes mellitus (11), and arteriosclerosis (12) have been disclosed. However, no study has reported on effects of grape seed extract on adiponectin. We investigated relationships between the intake of grape seed extract and adiponectin level (Study 2).

\section{MATERIALS AND METHODS}

Test foods. Table 1 shows compositions of the test tablets. Tablets including grape seed extract were prepared so that they were indistinguishable by their outer appearance, and either contained no proanthocyanidin (control), $200 \mathrm{mg}$ proanthocyanidin (200 $\mathrm{mg}$ group), or $400 \mathrm{mg}$ proanthocyanidin (400 $\mathrm{mg}$ group).

Subjects. Experimental protocols were approved by the Aiwa Clinic Institutional Review Board (KKM-001- 
Table 1. Composition of test tablets.

Group

Component

\begin{tabular}{ccc} 
& Group \\
Placebo & $\begin{array}{c}200 \mathrm{mg} / \mathrm{d} \\
(50 \mathrm{mg} \times 4 \text { tablets })\end{array}$ & $\begin{array}{c}400 \mathrm{mg} / \mathrm{d} \\
(100 \mathrm{mg} \times 4 \text { tablets })\end{array}$ \\
\hline- & 69.375 & 138.750 \\
- & $(50)$ & $(100)$ \\
0.025 & - & - \\
187.500 & 123.125 & 16.500 \\
54.975 & 50.000 & 7.500 \\
7.500 & 7.500 & trace amounts
\end{tabular}

Table 2. Clinical data.

\begin{tabular}{|c|c|c|c|c|}
\hline Item & Unit & Placebo & $200 \mathrm{mg}$ & $400 \mathrm{mg}$ \\
\hline Sex & $\mathrm{M} / \mathrm{F}$ & $10 / 10$ & $10 / 11$ & 9/11 \\
\hline Age & Year & $53.2 \pm 2.1$ & $51.0 \pm 2.4$ & $52.9 \pm 2.0$ \\
\hline Height & $\mathrm{cm}$ & $161 \pm 1.8$ & $161 \pm 2.0$ & $160 \pm 1.8$ \\
\hline Body weight & $\mathrm{kg}$ & $63.1 \pm 2.18$ & $63.5 \pm 2.68$ & $62.3 \pm 2.47$ \\
\hline BMI & & $24.4 \pm 0.59$ & $24.2 \pm 0.66$ & $24.1 \pm 0.62$ \\
\hline Systolic blood pressure & $\mathrm{mmHg}$ & $126.4 \pm 4.5$ & $127.0 \pm 3.2$ & $125.2 \pm 3.1$ \\
\hline Diastolic blood pressure & $\mathrm{mmHg}$ & $78.6 \pm 2.5$ & $76.5 \pm 1.8$ & $79.1 \pm 2.0$ \\
\hline Pulse & bpm & $65.3 \pm 1.3$ & $64.9 \pm 2.4$ & $67.8 \pm 1.9$ \\
\hline White blood cell count & $/ \mu \mathrm{L}$ & $5725 \pm 273$ & $6100 \pm 366$ & $5975 \pm 419$ \\
\hline Red blood cell count & $\times 10^{4} / \mu \mathrm{L}$ & $442 \pm 12$ & $448 \pm 9$ & $449 \pm 11$ \\
\hline Hemoglobin & $/ \mu \mathrm{L}$ & $13.7 \pm 0.31$ & $13.9 \pm 0.31$ & $13.9 \pm 0.31$ \\
\hline Hematocrit & $\%$ & $40.8 \pm 1.01$ & $41.4 \pm 0.93$ & $41.5 \pm 1.03$ \\
\hline Platelet count & $\times 10^{4} / \mu \mathrm{L}$ & $25.3 \pm 0.87$ & $24.5 \pm 0.99$ & $25.1 \pm 1.38$ \\
\hline LDL-Cholesterol & $\mathrm{mg} / \mathrm{dL}$ & $133 \pm 5.0$ & $134 \pm 3.7$ & $133 \pm 3.5$ \\
\hline HDL-Cholesterol & $\mathrm{mg} / \mathrm{dL}$ & $58 \pm 2.6$ & $63 \pm 3.2$ & $65 \pm 2.9$ \\
\hline Total cholesterol & $\mathrm{mg} / \mathrm{dL}$ & $224 \pm 5.7$ & $225 \pm 5.3$ & $225 \pm 4.4$ \\
\hline Triglycerides & $\mathrm{mg} / \mathrm{dL}$ & $135 \pm 19.3$ & $116 \pm 11.9$ & $119 \pm 14.0$ \\
\hline Total protein & $\mathrm{mg} / \mathrm{dL}$ & $7.3 \pm 0.10$ & $7.3 \pm 0.10$ & $7.3 \pm 0.09$ \\
\hline Albumin & $\mathrm{mg} / \mathrm{dL}$ & $4.4 \pm 0.05$ & $4.4 \pm 0.05$ & $4.6 \pm 0.05$ \\
\hline $\mathrm{A} / \mathrm{G}$ & $\mathrm{mg} / \mathrm{dL}$ & $1.6 \pm 0.03$ & $1.6 \pm 0.05$ & $1.7 \pm 0.04$ \\
\hline Total bilirubin & $\mathrm{mg} / \mathrm{dL}$ & $0.77 \pm 0.05$ & $0.68 \pm 0.04$ & $0.73 \pm 0.04$ \\
\hline AST (GOT) & $\mathrm{IU} / \mathrm{L} / 37^{\circ} \mathrm{C}$ & $22.0 \pm 1.97$ & $20.2 \pm 1.14$ & $23.1 \pm 1.54$ \\
\hline ALT (GPT) & $\mathrm{IU} / \mathrm{L} / 37^{\circ} \mathrm{C}$ & $25.3 \pm 2.84$ & $25.8 \pm 3.45$ & $27.9 \pm 2.41$ \\
\hline ALP & $\mathrm{IU} / \mathrm{L} / 37^{\circ} \mathrm{C}$ & $214 \pm 12.3$ & $230 \pm 14.3$ & $212 \pm 13.2$ \\
\hline $\mathrm{LDH}$ & $\mathrm{IU} / \mathrm{L} / 37^{\circ} \mathrm{C}$ & $171 \pm 5.4$ & $179 \pm 4.5$ & $172 \pm 6.4$ \\
\hline$\gamma$-GTP & $\mathrm{IU} / \mathrm{L} / 37^{\circ} \mathrm{C}$ & $38.1 \pm 7.33$ & $29.3 \pm 4.87$ & $33.6 \pm 4.59$ \\
\hline CPK & $\mathrm{IU} / \mathrm{L} / 37^{\circ} \mathrm{C}$ & $108 \pm 7.6$ & $108 \pm 8.7$ & $115 \pm 9.3$ \\
\hline Uric acid & $\mathrm{mg} / \mathrm{dL}$ & $5.6 \pm 0.31$ & $5.2 \pm 0.26$ & $5.2 \pm 0.33$ \\
\hline Blood urea nitrogen & $\mathrm{mg} / \mathrm{dL}$ & $13.5 \pm 0.62$ & $14.5 \pm 0.77$ & $12.7 \pm 0.50$ \\
\hline Creatinine & $\mathrm{mg} / \mathrm{dL}$ & $0.76 \pm 0.03$ & $0.73 \pm 0.03$ & $0.69 \pm 0.03$ \\
\hline Glucose & $\mathrm{mg} / \mathrm{dL}$ & $101 \pm 2.9$ & $96 \pm 1.7$ & $98 \pm 1.7$ \\
\hline $\mathrm{HbA}_{1 \mathrm{c}}$ & $\%$ & $4.94 \pm 0.10$ & $5.00 \pm 0.07$ & $5.03 \pm 0.08$ \\
\hline
\end{tabular}

Data represent means $\pm \mathrm{SE}$.

01). In compliance with the fundamental principles stipulated in the Helsinki Declaration, adequate explanations of the objectives and methods of this study were given to participants by attending physicians, and written consent forms were obtained from all enrolled subjects. The study period was from August to December 2003.

Subjects were selected to participate in the study if:

1) they were aged $\geq 30 \mathrm{y}$ and $<70 \mathrm{y} ; 2$ ) their LDL-C levels at baseline were $\geq 100 \mathrm{mg} / \mathrm{dL}$ and $<180 \mathrm{mg} / \mathrm{dL}$; 3) they discontinued drinking alcoholic beverages $2 \mathrm{~d}$ prior to the test; 4) they were given adequate explanations of the objectives and contents of the study, and provided written consent and voluntary wishes to participate in this study; and 5) they were attended by registered physicians.

Subjects were excluded if:

1) they were taking medicines that could affect lipid 
metabolism such as anti-hyperlipidemic drugs or antihypertensive drugs; 2) they were taking health foods suspected of affecting lipids or blood pressure; 3) they were commonly taking foods derived from grape seeds known to exert strong antioxidant effects (e.g., seed oil), health foods, or vitamin preparations (C, E) (including persons drinking lots of healthy tea); 4) they usually drank red wine (more than 1 half-bottle a week); 5) they had diabetes mellitus or remarkable hepatic dysfunctions; 6) they suffered from familial hyperlipidemia; 7 ) they were or were suspected of being pregnant, or were nursing mothers; 8) they were judged inappropri- ate by attending physicians.

Study methods. Following screening of 130 candidates, only 61 subjects were eligible for the study. Eligible subjects were randomly classified into 3 groups by the controller (Yoko Shoji, Associate Professor, St. Marianna University School of Medicine). A pre-observation period of $2 \mathrm{wk}$ preceded the 12-wk ingestion period, and the follow-up period was of $2 \mathrm{wk}$. Subjects ingested 4 tablets daily before breakfast every day for 12 wk. In cases with no breakfast, tablets were taken after lunch. Choice of tablet contents was left to the discretion of the subjects themselves. Both efficacy and

Table 3. Effects of grape seed extract supplementation on serum lipid concentration.

\begin{tabular}{|c|c|c|c|c|c|}
\hline Item & Group & $n$ & Before & Week 6 & Week 12 \\
\hline \multirow{3}{*}{$\begin{array}{l}\text { Total cholesterol } \\
\qquad(\mathrm{mg} / \mathrm{dL})\end{array}$} & Placebo & 18 & $219 \pm 4.9$ & $231 \pm 6.0^{\#}$ & $226 \pm 5.1^{\#}$ \\
\hline & $200 \mathrm{mg}$ & 18 & $226 \pm 7.1$ & $228 \pm 7.9$ & $225 \pm 7.7$ \\
\hline & $400 \mathrm{mg}$ & 17 & $222 \pm 4.9$ & $221 \pm 6.0$ & $229 \pm 7.4$ \\
\hline \multirow{3}{*}{$\begin{array}{l}\text { LDL-Cholesterol } \\
\qquad(\mathrm{mg} / \mathrm{dL})\end{array}$} & Placebo & 18 & $132 \pm 4.6$ & $143 \pm 5.3^{\#}$ & $138 \pm 4.5$ \\
\hline & $200 \mathrm{mg}$ & 18 & $134 \pm 4.9$ & $138 \pm 5.7$ & $134 \pm 5.4$ \\
\hline & $400 \mathrm{mg}$ & 17 & $132 \pm 4.4$ & $132 \pm 5.0$ & $138 \pm 6.1$ \\
\hline \multirow{3}{*}{$\begin{array}{l}\text { HDL-Cholesterol } \\
\qquad(\mathrm{mg} / \mathrm{dL})\end{array}$} & Placebo & 18 & $58.3 \pm 3.08$ & $61.4 \pm 2.92$ & $64.2 \pm 3.27^{\#}$ \\
\hline & $200 \mathrm{mg}$ & 18 & $63.1 \pm 4.10$ & $64.9 \pm 4.79$ & $68.7 \pm 4.86^{\#}$ \\
\hline & $400 \mathrm{mg}$ & 17 & $61.4 \pm 2.98$ & $63.6 \pm 3.18$ & $67.1 \pm 3.51^{\#}$ \\
\hline \multirow{3}{*}{$\begin{array}{l}\text { Triglycerides } \\
(\mathrm{mg} / \mathrm{dL})\end{array}$} & Placebo & 18 & $117 \pm 14.4$ & $132 \pm 18.1$ & $122 \pm 14.1$ \\
\hline & $200 \mathrm{mg}$ & 18 & $116 \pm 10.7$ & $148 \pm 22.2$ & $111 \pm 14.5$ \\
\hline & $400 \mathrm{mg}$ & 17 & $137 \pm 20.9$ & $120 \pm 14.7$ & $129 \pm 19.5$ \\
\hline \multirow{3}{*}{$\begin{array}{l}\text { RLP-Cholesterol } \\
\qquad(\mathrm{mg} / \mathrm{dL})\end{array}$} & Placebo & 18 & $6.4 \pm 1.24$ & $7.2 \pm 1.19$ & $6.0 \pm 1.00$ \\
\hline & $200 \mathrm{mg}$ & 18 & $6.0 \pm 0.45$ & $6.9 \pm 0.82$ & $5.2 \pm 0.63^{\#}$ \\
\hline & $400 \mathrm{mg}$ & 17 & $6.7 \pm 1.18$ & $5.7 \pm 0.66$ & $6.2 \pm 1.21$ \\
\hline \multirow{3}{*}{$\begin{array}{l}\text { Apolipoprotein A1 } \\
\text { (mg/dL) }\end{array}$} & Placebo & 18 & $145 \pm 4.3$ & $147 \pm 4.5$ & $147 \pm 3.9$ \\
\hline & $200 \mathrm{mg}$ & 18 & $153 \pm 5.9$ & $154 \pm 6.9$ & $154 \pm 6.0$ \\
\hline & $400 \mathrm{mg}$ & 17 & $151 \pm 4.2$ & $152 \pm 4.7$ & $153 \pm 5.3$ \\
\hline \multirow{3}{*}{$\begin{array}{l}\text { Apolipoprotein B } \\
\text { (mg/dL) }\end{array}$} & Placebo & 18 & $113 \pm 3.8$ & $115 \pm 4.4$ & $111 \pm 3.7$ \\
\hline & $200 \mathrm{mg}$ & 18 & $116 \pm 3.5$ & $113 \pm 3.5$ & $111 \pm 3.3^{\#}$ \\
\hline & $400 \mathrm{mg}$ & 17 & $115 \pm 3.1$ & $111 \pm 3.4^{\#}$ & $112 \pm 3.9$ \\
\hline \multirow{3}{*}{$\begin{array}{l}\text { Apolipoprotein E } \\
\quad(\mathrm{mg} / \mathrm{dL})\end{array}$} & Placebo & 18 & $4.4 \pm 0.38$ & $4.7 \pm 0.45^{\#}$ & $4.6 \pm 0.35$ \\
\hline & $200 \mathrm{mg}$ & 18 & $4.3 \pm 0.20$ & $4.4 \pm 0.22$ & $4.3 \pm 0.22$ \\
\hline & $400 \mathrm{mg}$ & 17 & $4.1 \pm 0.17$ & $4.2 \pm 0.21$ & $4.3 \pm 0.17$ \\
\hline \multirow[t]{3}{*}{ Apolipoprotein B/A1 } & Placebo & 18 & $0.80 \pm 0.04$ & $0.80 \pm 0.04$ & $0.77 \pm 0.03$ \\
\hline & $200 \mathrm{mg}$ & 18 & $0.78 \pm 0.04$ & $0.76 \pm 0.04$ & $0.74 \pm 0.04^{\#}$ \\
\hline & $400 \mathrm{mg}$ & 17 & $0.77 \pm 0.03$ & $0.74 \pm 0.03$ & $0.75 \pm 0.03$ \\
\hline \multirow{3}{*}{$\begin{array}{l}\text { MDA-LDL } \\
\quad(\mathrm{U} / \mathrm{L})\end{array}$} & Placebo & 18 & $122 \pm 7.0$ & $129 \pm 8.6$ & $113 \pm 7.4$ \\
\hline & $200 \mathrm{mg}$ & 18 & $122 \pm 6.4$ & $130 \pm 6.8$ & $107 \pm 5.3^{\#}$ \\
\hline & $400 \mathrm{mg}$ & 17 & $122 \pm 7.9$ & $108 \pm 6.3^{\#}$ & $105 \pm 6.5^{\#}$ \\
\hline \multirow{3}{*}{$\begin{array}{l}\text { MDA-LDL/Apo B } \\
(\mathrm{mU} / \mathrm{mg})\end{array}$} & Placebo & 18 & $107 \pm 3.7$ & $111 \pm 4.6$ & $102 \pm 5.8$ \\
\hline & $200 \mathrm{mg}$ & 18 & $105 \pm 4.5$ & $115 \pm 5.1$ & $97 \pm 4.1$ \\
\hline & $400 \mathrm{mg}$ & 17 & $105 \pm 5.0$ & $97 \pm 3.8^{\#}$ & $93 \pm 4.3^{\#}$ \\
\hline
\end{tabular}

Data represent means \pm SE.

\# Compared to before $(p<0.05)$. 
safety were evaluated 6 and 12 wk after the commencement of administration.

In Study 1, antioxidant effects and safety induced by grape seed extract were assessed, while in Study 2, effects of grape seed extract on adiponectin were investigated.

Items for determination. Height·body weight·body mass index (BMI), blood pressure-pulse rate, medical diagnosis findings. For blood biochemistry tests, total cholesterol, LDL-C, HDL-C, triglycerides, white blood cell counts, hematocrit, hemoglobin, platelet counts, total protein, albumin, A/G, total bilirubin, AST (GOT), ALT (GPT), ALP, LDH, $\gamma$-GTP, CPK, uric acid, BUN, creatinin, fasting blood glucose level, hemoglobin ${ }_{\mathrm{Alc}}\left(\mathrm{HbA}_{1 \mathrm{c}}\right)$, malondialdehyde modified LDL (MDA-LDL or oxidized LDL) as the oxidized LDL, remnant-like particles-cholesterol

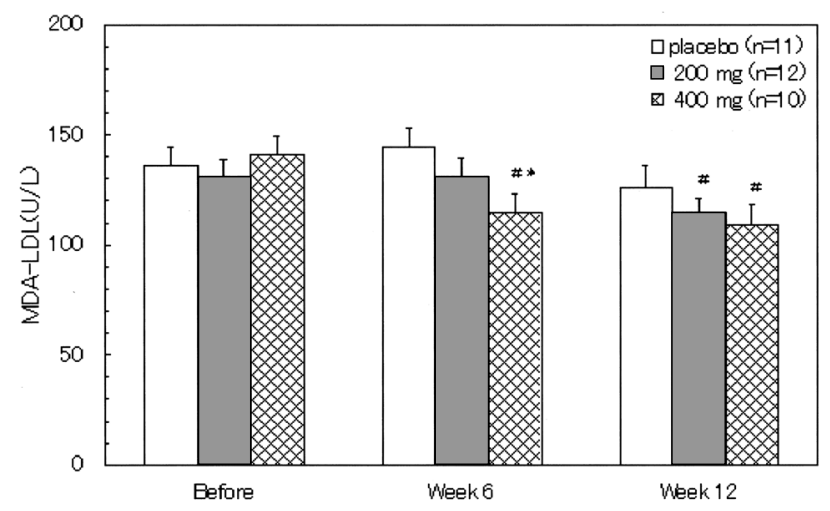

Fig. 1. Effects of grape seed extract supplementation on serum MDA-LDL in high MDA-LDL/ApoB subjects. Data represent means \pm SE. ${ }^{*} p<0.05$ compared to placebo using the Dunnett test. ${ }^{\#} p<0.05$ compared to before treatment using a paired $t$-test.
(RLP-C), apoprotein (immunoturbidimetric method), and adiponectin (ELISA method) were assessed. Urine tests were used to assess protein, glucose, occult blood reaction, and urine sediments. MDA-LDL levels were determined by an EIA method (13) while RLP-C was measured by immunoadsorption.

Statistical analysis. For Study 1, a paired $t$-test was used for within-group comparisons of antioxidant effects of grape seed extract and its safety evaluation while a one-way analysis of variance followed by a multiple comparison test (Dunnett test) was used for intergroup comparisons. With regards to MDA-LDL/ApoB, Kondo et al. (14) demonstrated that MDA-LDL/ApoB in subjects with low HDL (TG $\leq 1.69 \mathrm{mM}, \mathrm{HDL} \leq 0.91 \mathrm{~mm})$ was $99 \pm 49 \mathrm{mU} / \mathrm{mL}$; therefore, we called the group with more than $100 \mathrm{mU} / \mathrm{mL}$ MDA-LDL/ApoB the high MDA-LDL/ApoB group.

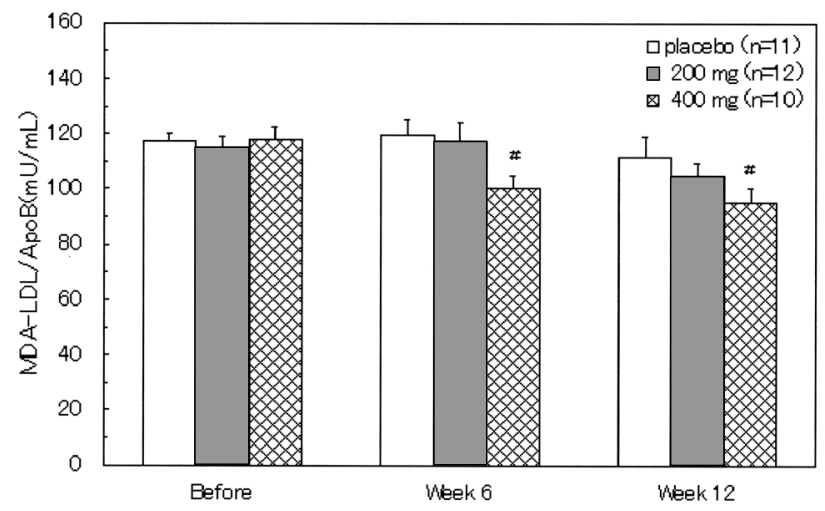

Fig. 2 Effects of grape seed extract supplementation on MDA-LDL/ApoB in high MDA-LDL/ApoB subjects. Data represent means \pm SE. ${ }^{*} p<0.05$ compared to before treatment using a paired $t$-test.

Table 4. Changes in body weight, BMI, blood pressure, pulse of subjects given grape seed extracts.

\begin{tabular}{llllll}
\hline \multicolumn{1}{c}{ Item } & Group & $n$ & Before & Week 6 & Week 12 \\
\hline $\begin{array}{l}\text { Body weight } \\
(\mathrm{kg})\end{array}$ & Placebo & 18 & $62.1 \pm 2.22$ & $62.0 \pm 2.19$ & $62.5 \pm 2.24$ \\
& $200 \mathrm{mg}$ & 18 & $63.5 \pm 2.71$ & $64.0 \pm 2.71$ & $64.1 \pm 2.70$ \\
& $400 \mathrm{mg}$ & 17 & $62.3 \pm 2.57$ & $62.8 \pm 2.56$ & $62.4 \pm 2.59$ \\
\hline BMI & Placebo & 18 & $24.2 \pm 0.63$ & $24.2 \pm 0.61$ & $24.3 \pm 0.64$ \\
& $200 \mathrm{mg}$ & 18 & $24.4 \pm 0.66$ & $24.6 \pm 0.66$ & $24.6 \pm 0.65$ \\
& $400 \mathrm{mg}$ & 17 & $24.0 \pm 0.65$ & $24.2 \pm 0.65$ & $24.1 \pm 0.64$ \\
\hline Systolic blood pressure & Placebo & 18 & $122.7 \pm 4.39$ & $124.2 \pm 4.99$ & $127.8 \pm 4.84$ \\
$(\mathrm{mmHg})$ & $200 \mathrm{mg}$ & 18 & $126.4 \pm 2.97$ & $127.8 \pm 3.22$ & $129.2 \pm 3.22$ \\
& $400 \mathrm{mg}$ & 17 & $126.2 \pm 3.98$ & $126.9 \pm 3.40$ & $127.7 \pm 3.00$ \\
\hline Diastolic blood pressure & Placebo & 18 & $77.1 \pm 2.60$ & $77.0 \pm 2.87$ & $81.1 \pm 2.80$ \\
$(\mathrm{mmHg})$ & $200 \mathrm{mg}$ & 18 & $77.9 \pm 1.66$ & $80.1 \pm 2.08$ & $79.6 \pm 2.00$ \\
& $400 \mathrm{mg}$ & 17 & $78.0 \pm 2.46$ & $79.1 \pm 2.39$ & $79.5 \pm 1.98$ \\
\hline Pulse & Placebo & 18 & $67.9 \pm 2.15$ & $66.5 \pm 2.44$ & $68.8 \pm 2.33$ \\
(bpm) & $200 \mathrm{mg}$ & 18 & $71.1 \pm 2.40$ & $70.6 \pm 2.62$ & $70.0 \pm 2.40$ \\
& $400 \mathrm{mg}$ & 17 & $72.8 \pm 2.28$ & $70.1 \pm 1.83$ & $73.0 \pm 2.54$
\end{tabular}

Data represent means \pm SE. 
Table 5. Changes in biochemical conditions of subjects given grape seed extracts.

\begin{tabular}{|c|c|c|c|c|c|c|}
\hline Item & Standard value & Group & $n$ & Before & Week 6 & Week 12 \\
\hline \multirow[t]{3}{*}{ White blood cell count } & 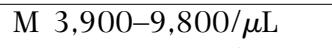 & Placebo & 18 & $5389 \pm 217$ & $5350 \pm 225$ & $5444 \pm 211$ \\
\hline & F $3,500-9,100 / \mu \mathrm{L}$ & $200 \mathrm{mg}$ & 18 & $5989 \pm 385$ & $5833 \pm 409$ & $5839 \pm 320$ \\
\hline & & $400 \mathrm{mg}$ & 17 & $5882 \pm 439$ & $5718 \pm 592$ & $5782 \pm 452$ \\
\hline \multirow[t]{3}{*}{ Red blood cell count } & $\mathrm{M} 427-570 \times 10^{4} / \mu \mathrm{L}$ & Placebo & 18 & $435 \pm 12$ & $447 \pm 12$ & $438 \pm 13$ \\
\hline & $\mathrm{F} \quad 376-500 \times 10^{4} / \mu \mathrm{L}$ & $200 \mathrm{mg}$ & 18 & $443 \pm 11$ & $453 \pm 11$ & $453 \pm 10$ \\
\hline & & $400 \mathrm{mg}$ & 17 & $449 \pm 13$ & $450 \pm 11$ & $454 \pm 11$ \\
\hline \multirow[t]{3}{*}{ Hemoglobin } & M $13.5-17.6 \mathrm{~g} / \mathrm{dL}$ & Placebo & 18 & $13.6 \pm 0.33$ & $14.1 \pm 0.35$ & $13.8 \pm 0.35$ \\
\hline & F $\quad 11.3-15.2 \mathrm{~g} / \mathrm{dL}$ & $200 \mathrm{mg}$ & 18 & $13.7 \pm 0.38$ & $14.0 \pm 0.36$ & $13.9 \pm 0.34$ \\
\hline & & $400 \mathrm{mg}$ & 17 & $13.9 \pm 0.36$ & $14.0 \pm 0.31$ & $14.1 \pm 0.31$ \\
\hline \multirow[t]{3}{*}{ Hematocrit } & M 39.8-51.8\% & Placebo & 18 & $40.6 \pm 1.06$ & $41.9 \pm 1.10$ & $41.1 \pm 1.20$ \\
\hline & F $\quad 33.4-44.9 \%$ & $200 \mathrm{mg}$ & 18 & $40.8 \pm 1.13$ & $41.8 \pm 1.09$ & $41.8 \pm 1.04$ \\
\hline & & $400 \mathrm{mg}$ & 17 & $41.5 \pm 1.20$ & $41.7 \pm 1.06$ & $42.0 \pm 1.07$ \\
\hline \multirow[t]{3}{*}{ Platelet count } & M $13.1-36.2 \times 10^{4} / \mu \mathrm{L}$ & Placebo & 18 & $24.1 \pm 0.98$ & $24.9 \pm 0.75$ & $24.8 \pm 0.93$ \\
\hline & F $\quad 13.0-36.9 \mathrm{l} \times 10^{4} / \mu \mathrm{L}$ & $200 \mathrm{mg}$ & 18 & $24.5 \pm 0.99$ & $25.8 \pm 1.13$ & $26.0 \pm 1.15$ \\
\hline & & $400 \mathrm{mg}$ & 17 & $25.4 \pm 1.19$ & $25.1 \pm 1.30$ & $25.8 \pm 1.19$ \\
\hline \multirow[t]{3}{*}{ Total protein } & $6.7-8.3 \mathrm{~g} / \mathrm{dL}$ & Placebo & 18 & $7.2 \pm 0.07$ & $7.4 \pm 0.09$ & $7.3 \pm 0.11$ \\
\hline & & $200 \mathrm{mg}$ & 18 & $7.3 \pm 0.07$ & $7.4 \pm 0.07$ & $7.5 \pm 0.07$ \\
\hline & & $400 \mathrm{mg}$ & 17 & $7.2 \pm 0.09$ & $7.2 \pm 0.08$ & $7.4 \pm 0.10$ \\
\hline \multirow[t]{3}{*}{ Albumin } & $4.0-5.0 \mathrm{~g} / \mathrm{dL}$ & Placebo & 18 & $4.3 \pm 0.04$ & $4.4 \pm 0.04$ & $4.4 \pm 0.06$ \\
\hline & & $200 \mathrm{mg}$ & 18 & $4.4 \pm 0.05$ & $4.4 \pm 0.06$ & $4.5 \pm 0.04$ \\
\hline & & $400 \mathrm{mg}$ & 17 & $4.4 \pm 0.05$ & $4.4 \pm 0.05$ & $4.5 \pm 0.05$ \\
\hline \multirow[t]{3}{*}{$\mathrm{A} / \mathrm{G}$} & $1.2-2.0$ & Placebo & 18 & $1.5 \pm 0.04$ & $1.5 \pm 0.03$ & $1.5 \pm 0.04$ \\
\hline & & $200 \mathrm{mg}$ & 18 & $1.5 \pm 0.05$ & $1.5 \pm 0.05$ & $1.5 \pm 0.05$ \\
\hline & & $400 \mathrm{mg}$ & 17 & $1.6 \pm 0.05$ & $1.6 \pm 0.04$ & $1.6 \pm 0.05$ \\
\hline \multirow[t]{3}{*}{ Total bilirubin } & $0.2-1.0 \mathrm{mg} / \mathrm{dL}$ & Placebo & 18 & $0.8 \pm 0.05$ & $0.8 \pm 0.05$ & $0.8 \pm 0.06$ \\
\hline & & $200 \mathrm{mg}$ & 18 & $0.7 \pm 0.04$ & $0.7 \pm 0.05$ & $0.7 \pm 0.06$ \\
\hline & & $400 \mathrm{mg}$ & 17 & $0.7 \pm 0.06$ & $0.7 \pm 0.06$ & $0.6 \pm 0.05$ \\
\hline \multirow[t]{3}{*}{ AST (GOT) } & $10-40 \mathrm{IU} / \mathrm{L} / 37^{\circ} \mathrm{C}$ & Placebo & 18 & $24.4 \pm 2.94$ & $23.1 \pm 2.02$ & $27.1 \pm 3.21$ \\
\hline & & $200 \mathrm{mg}$ & 18 & $22.2 \pm 1.37$ & $23.3 \pm 1.02$ & $25.2 \pm 0.95$ \\
\hline & & $400 \mathrm{mg}$ & 17 & $23.5 \pm 1.41$ & $22.7 \pm 1.02$ & $22.1 \pm 1.07$ \\
\hline \multirow[t]{3}{*}{ ALT (GPT) } & $5-40 \mathrm{IU} / \mathrm{L} / 37^{\circ} \mathrm{C}$ & Placebo & 18 & $24.9 \pm 3.34$ & $24.9 \pm 3.56$ & $31.2 \pm 5.34$ \\
\hline & & $200 \mathrm{mg}$ & 18 & $25.7 \pm 3.72$ & $27.7 \pm 3.32$ & $28.8 \pm 2.86$ \\
\hline & & $400 \mathrm{mg}$ & 17 & $26.5 \pm 2.57$ & $25.6 \pm 2.34$ & $25.0 \pm 1.45$ \\
\hline \multirow[t]{3}{*}{ ALP } & $115-359 \mathrm{IU} / \mathrm{L} / 37^{\circ} \mathrm{C}$ & Placebo & 18 & $205 \pm 13$ & $221 \pm 13$ & $224 \pm 13$ \\
\hline & & $200 \mathrm{mg}$ & 18 & $231 \pm 12$ & $243 \pm 13$ & $243 \pm 15$ \\
\hline & & $400 \mathrm{mg}$ & 17 & $207 \pm 15$ & $214 \pm 16$ & $213 \pm 16$ \\
\hline \multirow[t]{3}{*}{$\mathrm{LDH}$} & $115-245 \mathrm{IU} / \mathrm{L} / 37^{\circ} \mathrm{C}$ & Placebo & 18 & $171 \pm 8$ & $170 \pm 6$ & $170 \pm 7$ \\
\hline & & $200 \mathrm{mg}$ & 18 & $179 \pm 4$ & $182 \pm 5$ & $181 \pm 5$ \\
\hline & & $400 \mathrm{mg}$ & 17 & $164 \pm 7$ & $162 \pm 7$ & $160 \pm 6$ \\
\hline \multirow[t]{3}{*}{ g-GTP } & $\mathrm{M} \leqq 70 \mathrm{IU} / \mathrm{L} / 37^{\circ} \mathrm{C}$ & Placebo & 18 & $34.9 \pm 7.22$ & $40.1 \pm 8.63$ & $41.5 \pm 8.53$ \\
\hline & $\mathrm{F} \leqq 30 \mathrm{IU} / \mathrm{L} / 37^{\circ} \mathrm{C}$ & $200 \mathrm{mg}$ & 18 & $27.8 \pm 4.58$ & $30.2 \pm 4.54$ & $30.6 \pm 4.65$ \\
\hline & & $400 \mathrm{mg}$ & 17 & $33.8 \pm 4.71$ & $33.6 \pm 5.17$ & $33.4 \pm 5.07$ \\
\hline \multirow[t]{3}{*}{ CPK } & $\mathrm{M} 57-197 \mathrm{IU} / \mathrm{L} / 37^{\circ} \mathrm{C}$ & Placebo & 18 & $261 \pm 152$ & $102 \pm 6$ & $99 \pm 11$ \\
\hline & $\mathrm{F} \quad 32-180 \mathrm{IU} / \mathrm{L} / 37^{\circ} \mathrm{C}$ & $200 \mathrm{mg}$ & 18 & $116 \pm 9$ & $123 \pm 10$ & $120 \pm 13$ \\
\hline & & $400 \mathrm{mg}$ & 17 & $124 \pm 16$ & $116 \pm 11$ & $107 \pm 9$ \\
\hline Uric acid & $\mathrm{M} 3.7-7.0 \mathrm{mg} / \mathrm{dL}$ & Placebo & 18 & $5.5 \pm 0.37$ & $5.7 \pm 0.39$ & $5.4 \pm 0.37$ \\
\hline & $\mathrm{F} \quad 2.5-7.0 \mathrm{mg} / \mathrm{dL}$ & $200 \mathrm{mg}$ & 18 & $5.3 \pm 0.23$ & $5.4 \pm 0.24$ & $5.4 \pm 0.24$ \\
\hline & & $400 \mathrm{mg}$ & 17 & $5.2 \pm 0.28$ & $5.0 \pm 0.30$ & $4.9 \pm 0.29$ \\
\hline Blood urea nitrogen & $6-20 \mathrm{mg} / \mathrm{dL}$ & Placebo & 18 & $12.6 \pm 0.80$ & $12.5 \pm 0.54$ & $13.0 \pm 0.73$ \\
\hline & & $200 \mathrm{mg}$ & 18 & $14.1 \pm 0.89$ & $13.8 \pm 0.73$ & $13.1 \pm 0.78$ \\
\hline & & $400 \mathrm{mg}$ & 17 & $12.8 \pm 0.69$ & $11.9 \pm 0.85$ & $12.6 \pm 0.75$ \\
\hline Creatinine & M $0.61-1.04 \mathrm{mg} / \mathrm{dL}$ & Placebo & 18 & $0.8 \pm 0.04$ & $0.8 \pm 0.05$ & $0.7 \pm 0.04$ \\
\hline & $\mathrm{F} \quad 0.47-0.79 \mathrm{mg} / \mathrm{dL}$ & $200 \mathrm{mg}$ & 18 & $0.8 \pm 0.06$ & $0.7 \pm 0.04$ & $0.7 \pm 0.03$ \\
\hline & & $400 \mathrm{mg}$ & 17 & $0.7 \pm 0.04$ & $0.7 \pm 0.03$ & $0.7 \pm 0.03$ \\
\hline Glucose & $70-109 \mathrm{mg} / \mathrm{dL}$ & Placebo & 18 & $97.8 \pm 3.05$ & $98.0 \pm 2.71$ & $100.3 \pm 3.32$ \\
\hline & & $200 \mathrm{mg}$ & 18 & $93.5 \pm 1.96$ & $96.1 \pm 3.04$ & $95.8 \pm 2.38$ \\
\hline & & $400 \mathrm{mg}$ & 17 & $94.2 \pm 1.39$ & $95.5 \pm 2.61$ & $96.9 \pm 2.01$ \\
\hline $\mathrm{HbA}_{1 \mathrm{c}}$ & $4.3-5.8 \%$ & Placebo & 18 & $4.9 \pm 0.11$ & $4.9 \pm 0.11$ & $4.9 \pm 0.11$ \\
\hline & & $200 \mathrm{mg}$ & 18 & $5.1 \pm 0.08$ & $5.0 \pm 0.08$ & $5.0 \pm 0.08$ \\
\hline & & $400 \mathrm{mg}$ & 17 & $5.1 \pm 0.08$ & $5.0 \pm 0.08$ & $5.0 \pm 0.09$ \\
\hline
\end{tabular}


For Study 2, a paired $t$-test was used to assess effects of grape seed extract on adiponectin while a one-way analysis of variance followed by a multiple comparison test (Dunnett test) was used for inter-group comparisons. Furthermore, values indicating a correlation between adiponectin and various measurements were standardized, and subjected to a single regression analysis. The standardization of the data was calculated by the ratio of the standard deviation for the difference in mean value and each value. Including the age, the mean value was adjusted to 0 in all items, and the standard deviation was adjusted to 1 . Therefore, it is considered that the effect of the unit can be avoided, when the correlation coefficient is calculated.

All measured values were shown as means \pm SE. Measured values were analyzed with SPSS ver 13.0J (SPSS Japan Inc.). Significant level was less than $5 \%$ in the two-tail test.

\section{RESULTS}

Table 2 shows baseline values (at the time of screening) of eligible subjects. No test item was significantly different among the 3 groups. Investigations were performed for 53 subjects following exclusion of 4 subjects including 2 drop-out cases due to their own voluntary discretion during the test period, 1 case who caught the common cold accompanied by diarrhea and headaches (no correlation with the tablets), 1 case who experienced asthmatic attacks (no correlation with the tablets) and another 4 subjects whose compliance with instructions was poor.

Study 1 (Antioxidant effect of grape seed extract)

Table 3 shows temporal changes in serum lipid after intake of grape seed extract. Although total cholesterol and LDL-C levels significantly increased compared to basal levels in the control group, there was no significant difference among the groups. HDL-C levels significantly increased compared to those at baseline in the control, $200 \mathrm{mg}$, and $400 \mathrm{mg}$ groups $12 \mathrm{wk}$ after the start of administration. However, no significant difference was found among groups. During the study period, triglyceride levels didn't significantly change. RLP-C level in the $200 \mathrm{mg}$ group significantly decreased compared to values prior to the 12th week; however, there was no significant difference in RLP-C level with that in the control group. ApoA1 level did not show any significant changes during the study period. While ApoB level in the $200 \mathrm{mg}$ and $400 \mathrm{mg}$ groups significantly decreased compared to baseline values, no significant change in the control group was found. Despite a significant increase in ApoE level at the 6th week in the control group, no significant difference was observed among other groups. ApoB/A1 level in the $200 \mathrm{mg}$ group significantly decreased at week 12 , but there was no significant difference compared to the control group.

MDA-LDL level $6 \mathrm{wk}$ after the start of administration was significantly decreased compared to previous levels in the $400 \mathrm{mg}$ group, and was significantly reduced from previous levels $12 \mathrm{wk}$ after the start of administration both in the $200 \mathrm{mg}$ and $400 \mathrm{mg}$ groups. There was
Table 6. Correlations between each measured value and adiponectin

\begin{tabular}{lrr}
\hline \multicolumn{1}{c}{ Item } & \multicolumn{1}{c}{$r$} & \multicolumn{1}{c}{$p$} \\
\hline Age & 0.352 & 0.010 \\
Body weight & -0.597 & $<0.001$ \\
BMI & -0.470 & $<0.001$ \\
LDL-Cholesterol & 0.052 & 0.714 \\
MDA-LDL & -0.152 & 0.276 \\
HDL-Cholesterol & 0.426 & 0.001 \\
Total cholesterol & 0.154 & 0.270 \\
Triglycerides & -0.368 & 0.007 \\
RLP-Cholesterol & -0.303 & 0.027 \\
Apolipoprotein A1 & 0.371 & 0.006 \\
Apolipoprotein B & -0.241 & 0.083 \\
Apolipoprotein E & -0.106 & 0.449 \\
Apolipoprotein B/A1 & -0.395 & 0.003 \\
\hline
\end{tabular}

$n=53$.

a significant decrease in MDA-LDL/ApoB level from values prior to weeks 6 and 12 in the $400 \mathrm{mg}$ group.

Figure 1 illustrates changes in serum MDA-LDL levels of subjects with a high MDA-LDL/ApoB level (11 cases in the Placebo group, 12 cases in the $200 \mathrm{mg}$ group, and 10 cases in the $400 \mathrm{mg}$ group) demonstrating more than $100 \mathrm{mU} / \mathrm{mL} \mathrm{MDA}-\mathrm{LDL} / \mathrm{ApoB}$. Figure 2 shows temporal changes in MDA-LDL/ApoB levels. Serum MDA-LDL levels at week 6 in the $400 \mathrm{mg}$ group were significantly lower than previous values in the control group while they were significantly lower than previous values at the 12 th week both in the $200 \mathrm{mg}$ and $400 \mathrm{mg}$ groups. In addition, a multiple comparison test revealed a decreasing trend in the 200 and $400 \mathrm{mg}$ groups compared to the control group. MDA-LDL/ApoB levels were significantly decreased at the 6th and 12 th wk in the $400 \mathrm{mg}$ group compared to previous values, indicating a decreasing trend relative to that in the control group.

\section{Safety of tablets}

Tables 4 and 5 show results obtained for physiological and clinical laboratory tests after commencement of administration in the control group, and the $200 \mathrm{mg}$ and $400 \mathrm{mg}$ groups. From physiological and hematological tests, neither abnormal values nor abnormal changes were observed, and no abnormalities in subjective or objective symptoms were noted. Even in urinary sedimentation, no problematic findings were found (data are not shown).

Study 2 (Correlation between grape seed extract and adiponectin)

There were no significant changes in adiponectin levels attributable to ingestion of grape seed extract. To correlate adiponectin level with various parameters before ingestion of the tablets, the observed values were standardized and subjected to a single regression analysis. Results obtained are shown in Table 6. Adiponectin level showed a significant positive correlation with age $(r=0.352)$, HDL-C $(r=0.426)$, and ApoA1 $(r=0.371)$, but showed a significant negative correlation with body 
Table 7. Effects of grape seed extract supplementation on serum adiponectin (change in body weight $< \pm 1.0 \mathrm{~kg}$ ).

\begin{tabular}{cccccc}
\hline Item & Group & $n$ & Before & Week 6 & Week 12 \\
\hline \multirow{2}{*}{ Adiponectin $(\mu \mathrm{g} / \mathrm{mL})$} & Placebo & 10 & $9.98 \pm 1.30$ & $10.91 \pm 1.39^{\#}$ & $10.77 \pm 1.39$ \\
& $200 \mathrm{mg}$ & 11 & $8.83 \pm 0.87$ & $9.13 \pm 0.80$ & $8.89 \pm 0.86$ \\
& $400 \mathrm{mg}$ & 14 & $8.70 \pm 1.06$ & $8.93 \pm 1.01$ & $9.44 \pm 1.06^{\$}$ \\
\hline
\end{tabular}

Data represent means \pm SE.

\# Compared to before $(p<0.05)$.

${ }^{\$}$ Compared to before $(p=0.088)$.

weight $(r=-0.597)$, BMI $(r=-0.470)$, triglycerides $(r=-0.368), \quad$ RLP-C $\quad(r=-0.303), \quad$ and $\mathrm{ApoB} / \mathrm{A} 1$ $(r=-0.395)$. Among these parameters, adiponectin demonstrated the highest correlation with body weight. Accordingly, an analysis was conducted to investigate effects of grape seed extract on adiponectin level using subjects with the least alterations in body weight during the study period (less than $\pm 1 \mathrm{~kg}$ ). Results obtained are shown in Table 7 . Intake of grape seed extract tended to increase adiponectin level $(p=0.088)$ at the 12 th week in the $400 \mathrm{mg}$ group compared to previous values.

\section{DISCUSSION}

In the present study, a parallel inter-group test using a single blind method for 53 subjects presenting LDL-C values $\geq 100 \mathrm{mg} / \mathrm{dL}$ and $<180 \mathrm{mg} / \mathrm{dL}$ was performed following administration of grape seed extract for 12 wk. In Study 1, we investigated both the effects and safety of grape seed extract ingestion on oxidized LDL. In Study 2, effects of grape seed extract on adiponectin level were evaluated.

It is well recognized that oxidized LDL plays a role in the onset of arteriosclerosis. The present study showed that administration of grape seed extract lowered MDALDL levels and MDA-LDL/ApoB values, indicating MDA modification of ApoB which is the major apolipoprotein in LDL-C. In particular, remarkable decreases in both MDA-LDL and MDA-LDL/ApoB levels were observed in subjects with high MDA-LDL/ApoB. According to a previous report (14) showing that MDA-LDL/ApoB was $99 \pm 49 \mathrm{mU} / \mathrm{mL}$ in subjects with low levels of HDL, we defined subjects with MDA-LDL/ApoB values of more than $100 \mathrm{mU} / \mathrm{mL}$ as high MDA-LDL/ApoB subjects, who were considered to be higher risk persons predisposed to arteriosclerosis. Results showed that grape seed extract significantly decreased the MDA-LDL level $6 \mathrm{wk}$ after the start of tablet administration in subjects with high MDA-LDL/ApoB in the $400 \mathrm{mg}$ group compared to previous values and the control group. There was a significant reduction in the MDA-LDL level $12 \mathrm{wk}$ after tablet administration in the $200 \mathrm{mg}$ and $400 \mathrm{mg}$ groups compared to previous values. The MDA-LDL/ ApoB level in subjects with high MDA-LDL/ApoB was significantly reduced at weeks 6 and 12 in the $400 \mathrm{mg}$ group compared to previous values. Although a decreasing trend was observed in the $200 \mathrm{mg}$ group compared to previous values, this was not statistically significant. Such a decreasing effect on oxidized LDL by grape seed extract demonstrated dose-dependency.

It has been reported (15) that intake of red wine is associated with a reduction of mortality, and with a decrease in morbidity from dementia. These effects may be due to effects of polyphenol contained in grape seeds on various lifestyle-related diseases. The main phenolic components of red wine are anthocyanins, catechins, resveratrol, phenolic acids, and the flavonols quercetin and myricetin $(16,17)$. The concentrations of these components in red wine can vary widely $(18-20)$. Several in vitro studies have shown an antioxidant effect of red wine and fractionated phenolic compounds on LDL oxidation (21-24). The results of the present study show that supplementation of thuman subjects with grape seed extract can inhibit oxidation of LDL.

Proanthocyanidin, adopted as the major ingredient of the grape seed extract tablets, is contained in various plants, besides being contained in small amounts in fruits like grapes, in barley, in beans, and in their processed foods. By ingesting these foods, most of us intake proanthocyanidin daily. Proanthocyanidin is known to exert remarkable antioxidant effects in aqueous systems (25). Proanthocyanidin is specifically accumulated in blood vessel walls, and captures active oxygen in the interstitial fluid (aqueous system) of blood vessel walls, whereby generation of oxidized LDL is inhibited to reduce foam cells, leading to inhibition of the onset of arteriosclerosis (26). In fact, it is known that proanthocyanidin contained in grape seeds exhibits arteriosclerosis-inhibiting effects in rabbits fed with cholesterolloaded food (27). It was also reported that meals with concomitant ingestion of grape seed extract increased antioxidant substance levels in plasma, thereby reducing oxidized substances, resulting in decreasing postprandial oxidation-related stress, and increasing resistance of LDL toward oxidative modification (28-30). Preuss et al. (31) evaluated oxidated LDL autoantibodies as an index of reduction of other oxidized LDL. As a result, they reported that administration of grape seed extract to hyperlipidemic patients for $8 \mathrm{wk}$ decreased oxidized LDL autoantibody levels. No report is available on the possible effects of ingestion of grape seed extract for more than $12 \mathrm{wk}$ on oxidized LDL levels. Although there is no report on effects of grape seed extract on serum lipids following administration of grape seed extract for more than $12 \mathrm{wk}$, it was documented that administration of grape seed extract for $8 \mathrm{wk}$ was not 
associated with any effects on total cholesterol, LDL-C, or triglycerides (31). The same was true in the present study, as there was no effect on these lipids. In the present study, indices for evaluation of reducing effects of grape seed extract on oxidized LDL levels were MDALDL and MDA-LDL/ApoB in which MDA represents the most notable lipid peroxidized product bound to the lysine residue in ApoB in the form of Schiff's base. As a result, it was confirmed that grape seed extract had oxidized LDL reducing effects, and grape seed extract might be useful in preventing lifestyle-related diseases such as arteriosclerosis.

In addition, a possible correlation of adiponectin with lifestyle-related diseases has been recently reported. It has been reported that adiponectin levels in human blood are decreased in obese persons (type 2 diabetics), and in adult patients with coronary artery diseases, indicating the importance of anti-arteriosclerotic effects (32). Cnop et al. (33) revealed a positive correlation between adiponectin and HDL-C with age, and negative correlations between adiponectin and BMI, waist/hip, and triglycerides. It is known that red wine intake and moderate alcohol consumption increase the adiponectin level (34-36). The present study failed to clearly show effects of grape seed extract on adiponectin in all subjects included in the analysis. However, it was postulated that adiponectin had the highest correlation with body weight, as it was affected by changes in body weight. Accordingly, analysis was performed for subjects showing less alteration in body weight during the study period as eligible subjects, resulting in observation of an increase in adiponectin level due to administration of grape seed extract. However, this increase was not statistically significant.

Recently, it has been reported (37) that oolong tea increases plasma adiponectin level in patients with coronary artery diseases, and similar effects could be expected for grape seed extract. Altogether, increasing expectations exist for future research taking into account the age of subjects for evaluation. Furthermore, it has been verified that blood flow improves the effects of grape seed extract (38); therefore, grape seed extract could be useful in preventing lifestyle-related diseases.

During the study period, no harmful event attributable to the tablets containing grape seed extract was observed. Even in clinical laboratory tests, any alterations including minor changes fell within basal level ranges, indicating the safety of tablets containing grape seed extracts.

In conclusion, tablets containing grape seed extract are safe, and tend to particularly lower oxidized LDL and elevate adiponectin level in subjects with higher levels of oxidized LDL, indicating that grape seed extract might be a useful food in preventing the occurrence of lifestyle-related diseases including arteriosclerosis.

\section{REFERENCES}

1) Renaud S, de Lorgeril M. 1992. Wine, alcohol, platelets, and the French paradox for coronary heart disease. Lan- cet 339: 1523-1526.

2) Frankel EN, Waterhouse AL, Kinsella JE. 1993. Inhibition of human LDL oxidation by resveratrol. Lancet $\mathbf{3 4 1}$ : 1103-1104.

3) Kondo K, Matsumoto A, Kurata H, Takahashi H, Koda T, Amachi T, Itakura H. 1994. Inhibition of oxidation of low-density lipoprotein with red wine. Lancet $\mathbf{3 4 4}$ : 1152.

4) Dixon RA, Xie DY, Sharma SB. 2005. Proanthocyanidins - a final frontier in flavonoid research? New Phytologist 165: 9-28

5) Tanahashi H, Kondo K, Suwa Y, Zenibayashi Y, Toyoda Y, Kitamura K, Hosoda K, Amachi T, Matsumoto A, Itakura H. 1995. Component and clinical studies on antioxidative activity of red wine. ASEV Jap Rep 6: 241

6) Steinberg D, Parthasarathy S, Carew TE, Khoo JC, Witztum JL. 1989. Modification of low density lipoprotein that increase its atherogenicity. N Engl J Med 320: 915924.

7) Witztum JL, Steinberg D. 1991. Role of oxidized low density lipoprotein in atherogenesis. J Clin Invest $\mathbf{8 8}$ : 1785-1792.

8) Berliner JA, Navab M, Fogelam AM, Frank JS, Demer LL, Edwards PA, Watson AD, Lusis AJ. 1995. Atherosclerosis: basic mechanism. Oxidation, inflammation, and genetics. Circulation 91: 2488-2496.

9) Navab M, Berliner JA, Watson AD, Hama SY, Territo MC, Lusis AJ, Shih DM, Van Lenten BJ, Frank JS, Demer LL, Edwards PA, Fogelman AM. 1996. The Yin and Yang of oxidation in the development of the fatty streak. A review based on the 1994 George Lyman Duff Memorial Lecture. Arterioscler Thromb Vasc Biol 16: 831-842.1)

10) Arita Y, Kihara S, Ouchi N, Takahashi M, Maeda K, Miyagawa J, Hotta K, Shimomura I, Nakamura T, Miyaoka K, Kuriyama H, Nishida M, Yamashita S, Okubo K, Matsubara K, Kuraguchi M, Ohmoto Y, Funabashi T, Matsuzawa Y. 1999. Paradoxical decrease of an adipose-specific protein, adiponectin, in obesity. Biochem Biophys Res Commun 257: 79-83.

11) Hotta K, Funahashi T, Arita Y, Takahashi M, Matsuda M, Okamoto Y, Iwahashi H, Kuriyama H, Ouchi N, Maeda K, Nishida M, Kihara S, Sakai N, Nakajima T, Hasegawa K, Muraguchi M, Ohmoto Y, Nakamura T, Yamashita S, Hanafusa T, Matsuzawa Y. 2000. Plasma concentrations of a novel, adipose-specific protein, adiponectin, in type 2 diabetic patients. Arterioscler Thromb Vasc Biol 20: 1595-1599.

12) Ouchi N, Kihara S, Arita Y, Maeda K, Kuriyama H, Okamoto Y, Hotta K, Nishida M, Takahashi M, Nakamura T, Yamashita S, Funabashi T, Matsuzawa Y. 1999. Novel modulator for endothelial adhesion molecules: adipocyte-derived plasma protein adiponectin. Circulation 100: $2473-2476$.

13) Kitano S, Kanno T, Maekawa M, Sakurabayashi I, Kotani K, Hisatomi H, Hibi N, Kubono K, Harada S. 2004. Improved method for the immunological detection of malondialdehyde-modified low-density lipoproteins in human serum. Anal Chem Acta 509: 229-235.

14) Kondo A, Li J, Manabe M, Saito K, Kanno T, Maekawa M. 2003. Relationship between high-density lipoprotein-cholesterol and malondialdehyde-modified lowdensity lipoprotein concentrations. J Atheroscler Thromb 10: $72-78$.

15) Orgogozo JM, Dartigues JF, Lafont S, Letenneur L, Commenges D, Salamon R, Renaud SC, Breteler MB. 1997. 
Wine consumption and dementia in the elderly: a prospective community study in the Bordeaux area. Rev Neurol 153: 185 -192.

16) Nigdikar SV, Williams NR, Griffin BA, Howard AN. 1996. Consumption of red wine reduces the susceptibility of low-density lipoproteins to oxidation in vivo. Am J Clin Nutr 68: 258-265.

17) Roggero JP, Archier P, Coen S. 1997. Chromatography of phenolics in wine. ACS Symposium Series 661: 6-11.

18) Goldberg DM, Tsang E, Karumanchiri A, Soleas GJ. 1998. Quercetin and $p$-coumaric acid concentrations in commercial wines. Am J Enol Vitic 49: 142-151.

19) Sato M, Ramarathan N, Suzuki Y, Ohkubo T, Takeuchi M, Ochi H. 1996. Varietal differences in the phenolic content and superoxide radical scavenging potential of wines from different sources. J Agric Food Chem 44: 3741.

20) Waterhouse AL, Teissedre PL. 1997. Levels of phenolics in California varietal wines. ACS Symposium Series 661: 12-23.

21) Frankel EN, Kanner J, German JB, Parks E, Kinsella JE. 1993. Inhibition of oxidation of human low-density lipoprotein by phenolic substances in red wine. Lancet 341: 454-457.

22) AbuAmsha R, Croft KD, Puddy IB, Proudfoot JM, Beilin LJ. 1996. Phenolic content of various beverages determines the extent of inhibition of human serum and lowdensity-lipoprotein oxidation in-vitro. Identification and mechanism of action of some cinnamic acid-derivatives from red wine. Clin Sci 91: 449-458.

23) Kerry NL, Abbey M. 1997. Red wine and fractionated phenolic compounds prepared from red wine inhibit low density lipoprotein oxidation in vitro. Atherosclerosis 135: 93-102.

24) Gardner PT, McPhail DB, Crozier A, Duthie GG. 1999. Electron spin resonance (ESR) spectroscopic assessment of the contribution of quercetin and other flavonols to the antioxidant capacity of red wine. J Sci Food Agric 79: 1011-1014.

25) Ariga T, Koshiyama I, Fukushima D. 1990. Radical scavenging action and its mode in procyanidins B- 1 and B-3 from azuki beans to peroxyl radicals. Agric Biol Chem 54: 2499.

26) Laparra J, Michaud J, Masquelier J. 1977. Pharmacokinetic study of flavanolic oligomers. J Plant Med Phytother 11: 133-142.

27) Yamakoshi J, Kataoka S, Koga T, Ariga T. 1999. Proanthocyanidin-rich extract from grape seeds attenuates the development of aortic atherosclerosis in cholesterol- fed rabbits. Atherosclerosis 142: 139-149.

28) Natella F, Ghiselli A, Guidi A, Ursini F, Scaccini C. 2001. Red wine mitigates the postprandial increase of LDL susceptibility to oxidation. Free Radic Biol Med 30: 10361044 .

29) Natella F, Belelli F, Gentili V, Ursini F, Scaccini C. 2002. Grape seed proanthocyanidins prevent plasma postprandial oxidative stress in humans. J Agric Food Chem 50: 7720-7725.

30) Ventura P, Bini A, Panini R, Marri L, Tonasi A, Salvioli G.. 2004. Red wine consumption prevents vascular oxidative stress induced by a high-fat meal in healthy volunteers. Int J Vitam Nutr Res 74: 137-143.

31) Preuss HG, Wallerstedt D, Talpur N, Tutuncuoglu SO, Echard B, Myers A, Bui M, Bagchi D. 2000. Effects of niacin-bound chromium and grape seed proanthocyanidin extract on the lipid profile of hypercholesterolemic subjects: a pilot study. J Med 31: 227-246.

32) Funahashi T, Matsuzawa Y. 2002. Adiponectin. Nippon Rinsho 60: 583-592.

33) Cnop M, Havel PJ, Utzschneider KM, Carr DB, Sinha MK, Boyko EJ, Retzlaff BM, Knopp RH, Brunzell JD, Kahn SE. 2003. Relationship of adiponectin to body fat distribution, insulin sensitivity and plasma lipoproteins: evidence for independent roles of age and sex. Diabetologia 46: 459-469.

34) Sierksma A, Patel H, Ouchi N, Kihara S, Funahashi T, Heine RJ, Grobbee DE, Kiuft C, Hendriks HFJ. 2004. Effect of moderate alcohol consumption on adiponectin, tumor necrosis factor- $\alpha$, and insulin sensitivity. Diabetes Care 27: 184-189.

35) Pischon T, Girman CJ, Rifai N, Hotamisligil GS, Rimm EB. 2005. Association between dietary factors and plasma adiponectin concentrations in men. Am J Clin Nutr 81: 780-786.

36) Beulens JW, van Beers RM, Stolk RP, Schaafsma G, Hendriks HF. 2006. The effect of moderate alcohol consumption on fat distribution and adipocytokines. Obesity 14: 60-66.

37) Shimada K, Kawarabayashi T, Tanaka A, Fukuda D, Nakamura Y, Yoshiyama M, Takeuchi K, Sawaki T, Hosoda K, Yoshikawa J. 2004. Oolong tea increases plasma adiponectin levels and low-density lipoprotein particle size patients with coronary artery disease. Diabetes Res Clin Pract 65: 227-234.

38) Yoshimura M, Saito M, Tokutake S, Kikuchi M, Kikuchi Y. 2002. The effect of grape seed extracts on the fluidity of whole blood through capillary-model microchannels. Hemorheol Related Res 5: 35-37. 